\title{
REPRESENTATIONS OF THE SUPER JORDAN PLANE
}

\author{
NICOLÁS ANDRUSKIEWITSCH, DIRCEU BAGIO, SARADIA DELLA FLORA, \\ DAIANA FLÔRES \\ To Antonio Paques.
}

\begin{abstract}
It is shown that the finite-dimensional simple representations of the super Jordan plane $\mathcal{B}$ are one-dimensional. The indecomposable representations of dimension 2 and 3 of $\mathcal{B}$ are classified. Two families of indecomposable representations of $\mathcal{B}$ of arbitrary dimension are presented.
\end{abstract}

\section{INTRODUCTION}

Nichols algebras are graded connected algebras with a comultiplication in a braided sense. In particular, the Jordan plane and the super Jordan plane are two Nichols algebras that play an important role in the classification of pointed Hopf algebras with finite Gelfand-Kirillov dimension AAH1, AAH2].

The Jordan plane was first defined in $[\mathrm{G}$ and considered in many papers, e.g. AS], see also the references in [AAH2, I]. Its representation theory was studied in [I].

The purpose of this note is to begin the study of the representation theory of the super Jordan plane $\mathcal{B}$ : we classify the simple finite-dimensional $\mathcal{B}$-modules (all of dimension 1 , Theorem 2.6) and the indecomposable $\mathcal{B}$ modules of dimension 2 (Theorem [3.2) and 3 (Theorem 3.11). We also observe that one of the generators of $\mathcal{B}$ has at most two eigenvalues in every indecomposable $\mathcal{B}$-module (Theorem 2.11) and describe two families of indecomposable modules in every dimension.

\section{BASIC FACTS}

2.1. Notations and conventions. Fix an algebraically closed field $\mathbb{k}$ of characteristic 0; all vector spaces, tensor products, Hom spaces, algebras are over $\mathbb{k}$. All algebras are associative and all modules are left, unless explicitly stated. Let $A$ be a $\mathbb{k}$-algebra; then [, ] denotes the Lie bracket given by the commutator. As customary we use indistinctly the languages of modules

2000 Mathematics Subject Classification. 16G30,16G60, 16 T05.

N. A. was partially supported by CONICET, Secyt (UNC) and the MathAmSud project GR2HOPF.

D. B. and D. F. were supported by FAPERGS 2193-25.51/13-3, MATHAMSUD. 
and representations. Denote by ${ }_{A} \mathcal{M}$ the category of finite dimensional $A$ modules. Given a $\mathbb{k}$-vector space $V, \mathfrak{g l}(V)$ denotes the Lie algebra of all linear operators on $V$. The Jacobson radical of an algebra $A$ it will be denoted by Jac A.

2.2. The Jordan plane. The Jordan plane is the free associative algebra $\mathcal{A}$ in generators $y_{1}$ and $y_{2}$ subject to the quadratic relation

$$
y_{1} y_{2}-y_{2} y_{1}-y_{2}^{2}
$$

The algebra $\mathcal{A}$ is a Nichols algebra, $\operatorname{GKdim} \mathcal{A}=2$ and $\left\{y_{1}^{a} y_{2}^{b}: a, b \in \mathbb{N}_{0}\right\}$ is a basis of $\mathcal{A}$. By Proposition 3.4 of [I], $\mathcal{A}$ is a Koszul algebra.

2.3. The super Jordan plane. Let $x_{21}=x_{1} x_{2}+x_{2} x_{1}$ in the free associative algebra in generators $x_{1}$ and $x_{2}$. Let $\mathcal{B}$ be the algebra generated by $x_{1}$ and $x_{2}$ with defining relations

$$
\begin{aligned}
& x_{1}^{2}, \\
& x_{2} x_{21}-x_{21} x_{2}-x_{1} x_{21} .
\end{aligned}
$$

The algebra $\mathcal{B}$ (which is graded by $\operatorname{deg} x_{1}=\operatorname{deg} x_{2}=1$ ) was introduced in $\mathrm{AAH1}, \mathrm{AAH2}$ and is called the super Jordan plane. Since $\mathcal{B}$ is not a quadratic algebra, it follows that $\mathcal{B}$ is not Koszul; see e. g. $\S 2.1$ of $[\mathrm{PP}]$.

Proposition 2.1. AAH2 The algebra $\mathcal{B}$ is a Nichols algebra, GKdim $\mathcal{B}=2$ and $\left\{x_{1}^{a} x_{21}^{b} x_{2}^{c}: a \in\{0,1\}, b, c \in \mathbb{N}_{0}\right\}$ is a basis of $\mathcal{B}$.

The following identities are valid in $\mathcal{B}$ :

$$
\begin{aligned}
x_{21} x_{1} & =x_{1} x_{21}, \\
x_{2}^{2} x_{1} & =x_{1} x_{2}^{2}+x_{1} x_{2} x_{1}, \\
x_{21} x_{2}^{2} & =\left(x_{2}^{2}-x_{21}\right) x_{21} .
\end{aligned}
$$

Indeed, in presence of (2.1), (2.2) is equivalent to (2.4).

By (2.5) and Proposition 2.1, the subalgebra of the super Jordan plane $\mathcal{B}$ generated by $x_{2}^{2}$ and $x_{21}$, is isomorphic to the Jordan plane via $y_{1} \mapsto x_{2}^{2}$ and $y_{2} \mapsto x_{21}$.

It is convenient to introduce $s=x_{21}$ and $t=x_{2}^{2}$. By (2.5), st $=t s-s^{2}$ and whence

$$
\left[t, s^{n}\right]=n s^{n+1}, n \geq 1 ; \quad x_{1} s=s x_{1} ; \quad x_{2} t=t x_{2} ; \quad t x_{1}=x_{1}(t+s) .
$$

Lemma 2.2. Given $b, c \in \mathbb{N}$, we have that

$$
x_{21}^{b} x_{2}^{c} \stackrel{*}{=}\left(x_{2}-b x_{1}\right) x_{21}^{b} x_{2}^{c-1}, \quad x_{1} x_{21}^{b} x_{2}^{c} \stackrel{\rho}{=} x_{1} x_{2} x_{21}^{b} x_{2}^{c-1} .
$$

Proof. We prove $*$ by induction. For $b=c=1$, the relation is valid by (2.2). Suppose that $*$ is valid for $b-1>0$ and $c=1$. Then

$$
\begin{aligned}
x_{21}^{b} x_{2} & =x_{21}^{b-1} x_{21} x_{2}=x_{21}^{b-1}\left(x_{2} x_{21}-x_{1} x_{21}\right) \\
& =\left(x_{2}-(b-1) x_{1}\right) x_{21}^{b-1} x_{21}-x_{21}^{b} x_{1}=\left(x_{2}-b x_{1}\right) x_{21}^{b} .
\end{aligned}
$$


Fix $b \in \mathbb{N}$ and assume that the relation is true for $c-1$, with $c>1$. Thus

$$
x_{21}^{b} x_{2}^{c}=\left(x_{21}^{b} x_{2}^{c-1}\right) x_{2}=\left(x_{2}-b x_{1}\right) x_{21}^{b} x_{2}^{c-2} x_{2}=\left(x_{2}-b x_{1}\right) x_{21}^{b} x_{2}^{c-1} .
$$

The proof of $\varnothing$ is similar.

The next result follows immediately from Proposition 2.1 and Lemma 2.2 .

Proposition 2.3. The set $\left\{1, x_{1}, x_{2}, x_{1} x_{2}\right\}$ generates $\mathcal{B}$ as a right $\mathcal{A}$-module.

2.4. Simple modules. Let $(V, \rho)$ be a finite-dimensional representation of $\mathcal{B}$; set $X_{1}=\rho\left(x_{1}\right), X_{2}=\rho\left(x_{2}\right), S=\rho(s)$ and $T=\rho(t)$ and

$$
V_{0}=\operatorname{ker} X_{1} \text {. }
$$

Then $V_{0}$ is always $\neq 0$ and it is stable under $S$ and $T$ by (2.6). In fact, let $E_{12}(n) \in \mathfrak{g l}\left(\mathbb{k}^{n}\right)$ (or $E_{12}$ if $n$ is clearly from de context) the matrix whose the entry $1 \times 2$ is equal to 1 and all other entries are equal to 0 . Then the Jordan form of $X_{1}$ consists of $r$ blocks like $E_{12}(2)$ and $s$ blocks of size 1 filled by 0 . Hence $\operatorname{dim} V=2 r+s ; r=0 \Longleftrightarrow V=V_{0}$.

Lemma 2.4. Assume the previous notations. Then:

(i) $S$ and $T$ have a simultaneous eigenvector in $V_{0}$.

(ii) $W=X_{2} V_{0} \cap V_{0}$ is a submodule of $V$.

(iii) $U=X_{2} V_{0}+V_{0}$ is a submodule of $V$.

Proof. (i) The subspace of $\mathfrak{g l}(V)$ generated by $T$ and $S^{n}, n \in \mathbb{N}_{0}$, is a solvable Lie subalgebra by (2.6); then Lie Theorem applies.

(ii) Clearly $X_{1} W \subseteq X_{1} V_{0}=\{0\} \subseteq W$. It remains to show that $X_{2} W \subseteq$ $W$. In fact, let $w \in W$, this is, $w \in V_{0}$ and $w=X_{2} v$ for some $v \in V_{0}$. Clearly $X_{2} w \in X_{2} V_{0}$. Moreover,

$$
X_{1}\left(X_{2} w\right)=X_{1} X_{2}^{2} v \stackrel{\sqrt{2.4}}{=}\left(X_{2}^{2} X_{1}-X_{1} X_{2} X_{1}\right) v=0 \Longrightarrow X_{2} w \in W .
$$

(iii) Since $\mathcal{B} \cdot V_{0} \subseteq X_{2} V_{0}$ and $\mathcal{B} \cdot\left(X_{2} V_{0}\right) \subseteq V_{0}$, the claim follows.

Lemma 2.5. If $V \in \in_{\mathcal{B}} \mathcal{M}$ is simple, then $V=V_{0}$.

Proof. Assume that $V \neq V_{0}$. By Lemma 2.4 we have that $W=X_{2} V_{0} \cap V_{0}=0$ and $V=X_{2} V_{0}+V_{0}$, so that $V=X_{2} V_{0} \oplus V_{0}$. By Lemma 2.4 (i), there exists a simultaneous eigenvector $v \in V_{0}$ of $S$ and $T$, i.e. there exist $\alpha, \tau \in \mathbb{k}$ such that $S v=\alpha v, T v=\tau v$.

Now $M=\operatorname{span}\left\{v, X_{2} v\right\} \neq 0$ is a $\mathcal{B}$-submodule of $V$ and by simplicity of $V, M=V$. By our assumption, $X_{2} v \notin V_{0}$; hence $\Lambda=\left\{v, X_{2} v\right\}$ is a basis of $V$. Note that $\left[X_{1}\right]_{\Lambda}=\left(\begin{array}{cc}0 & \alpha \\ 0 & 0\end{array}\right)$ and $\left[X_{2}\right]_{\Lambda}=\left(\begin{array}{ll}0 & \tau \\ 1 & 0\end{array}\right)$. The relation $X_{2}^{2} X_{1}=X_{1} X_{2}^{2}+X_{1} X_{2} X_{1}$ is satisfied if and only if $\tau \alpha=\alpha \tau+\alpha^{2}$. Therefore $\alpha=0$ and $V=V_{0}$, a contradiction.

Let $A \in \operatorname{End}\left(\mathbb{k}^{n}\right)$. Denote by $\mathbb{k}_{A}^{n}$ the $\mathcal{B}$-module defined by $X_{1}=0$ and $X_{2}=A$. Every $\mathcal{B}$-module $V$ with $V=V_{0}$ is isomorphic to $\mathbb{k}_{A}^{n}$ for some $A$. If $B \in \operatorname{End}\left(\mathbb{k}^{m}\right)$, then $\mathbb{k}_{A}^{n} \simeq \mathbb{k}_{B}^{m}$ iff $n=m$ and $A$ and $B$ are similar matrices. 
Theorem 2.6. Every simple $\mathcal{B}$-module is isomorphic to $\mathbb{k}_{a}^{1}$ for a unique $a \in \mathbb{k}$.

Proof. This follows from Lemma 2.5 and the preceding considerations.

Corollary 2.7. Let $\rho: \mathcal{B} \rightarrow$ End $V$ a finite dimensional representation of $\mathcal{B}$ and $B=\rho(\mathcal{B})$. Then there exists an integer $s$ such that $B / \mathrm{Jac} B \simeq \mathbb{K}^{s}$ and Jac $B=\{x \in B: x$ is nilpotent $\}$.

Proof. Since $B / \operatorname{Jac} B$ is semisimple and $\mathbb{k}$ is algebraically closed, there are positive integers $n_{1}, \ldots, n_{s}$ such that $B / \operatorname{Jac} B=M_{n_{1}}(\mathbb{k}) \times \cdots \times M_{n_{s}}(\mathbb{k})$. The composition

$$
\mathcal{B} \stackrel{\rho}{\longrightarrow} B \stackrel{\pi}{\longrightarrow} B / \mathrm{Jac} B \stackrel{\pi_{j}}{\longrightarrow} M_{n_{j}}(\mathbb{k})
$$

is a finite dimensional simple representation of $\mathcal{B}$. Hence, by Theorem (2.6), $n_{1}=\cdots=n_{s}=1$. Thus, $B / \operatorname{Jac} B \simeq \mathbb{k}^{s}$. Let $x \in B$ a nilpotent element. Then $\pi(x)$ is a nilpotent element of $B / \mathrm{Jac} B$. Since $B / \mathrm{Jac} B$ is commutative, we obtain that $\pi(x) \in \operatorname{Jac}(B / \operatorname{Jac} B)=\{0\}$. Hence, $x \in \operatorname{Jac} B$. On the other hand, $B$ finite dimensional implies that $\operatorname{Jac} B$ is a nilpotent ideal. Consequently, Jac $B=\{x \in B: x$ is nilpotent $\}$.

We also remark:

Proposition 2.8. If $V$ is an indecomposable $\mathcal{B}$-module with $V=V_{0}$, then there exist $n \in \mathbb{N}$ and $\lambda \in \mathbb{k}$ such that $V$ is isomorphic to $\mathbb{k}_{A}^{n}$ where $A$ is the Jordan block of size $n$ with eigenvalue $\lambda$.

If $A$ is the Jordan block of size $n$ with eigenvalue $\lambda$, then denote $\mathcal{A}_{\lambda}=\mathbb{k}_{A}^{n}$.

2.5. Indecomposable modules. Throughout this subsection, $V, X_{1}, X_{2}$, $T$ and $S$ are as in $\oint 2.4$. When $V$ is indecomposable, we will prove that $T$ has a unique eigenvalue. In order to do this, the following relations are useful.

Lemma 2.9. Let $\lambda \in \mathbb{k}, z:=t-\lambda \mathrm{id} \in \mathcal{B}$ and $n \in \mathbb{N}$. Then

$$
z^{n} x_{1} \stackrel{\text { 中 }}{=} x_{1} \sum_{j=0}^{n} \frac{n !}{(n-j) !} s^{j} z^{n-j}, \quad z^{n} x_{1} x_{2} \triangleq x_{1} x_{2} \sum_{j=0}^{n} \frac{n !}{(n-j) !} s^{j} z^{n-j} .
$$

Proof. We prove by induction on $n$; the proof of $\diamond$ is similar. We will use that $x_{1} z s^{n}=x_{1} x_{21}^{n} z+n x_{1} s^{n+1}$, which can be verified easily. Note that

$$
z x_{1}=x_{1} z+x_{1} x_{2} x_{1}=x_{1} z+x_{1} s=x_{1}(z+s),
$$


and whence the formula is true for $n=1$. Denote $\zeta_{n, j}:=\frac{n !}{(n-j) !}, 0 \leq j \leq n$. Consider $n>1$ and assume that the formula is true for $n-1$. Then

$$
\begin{aligned}
z^{n} x_{1}=\left(z x_{1}\right) \sum_{j=0}^{n-1} \zeta_{n-1, j} s^{j} z^{n-1-j} \stackrel{\sqrt{2.2}}{=}\left(x_{1} z+x_{1} s\right) \sum_{j=0}^{n-1} \zeta_{n-1, j} s^{j} z^{n-1-j} \\
=\sum_{j=0}^{n-1} \zeta_{n-1, j}\left(x_{1} z s^{j}\right) z^{n-1-j}+\sum_{j=0}^{n-1} \zeta_{n-1, j} x_{1} s^{j+1} z^{n-1-j}=x_{1} \sum_{j=0}^{n} \zeta_{n, j} s^{j} z^{n-j} .
\end{aligned}
$$

Let $\lambda$ be an eigenvalue of $T$. Denote by $V_{\lambda}^{T}$ the generalized eigenspace of $V$ associated to $\lambda$, i. e. $V_{\lambda}^{T}:=\cup_{j \geq 0} \operatorname{ker}(T-\lambda \mathrm{id})^{j}$

Lemma 2.10. $V_{\lambda}^{T}$ is a $\mathcal{B}$-submodule of $V$, for all eigenvalue $\lambda$ of $T$.

Proof. Clearly $V_{\lambda}^{T}=\operatorname{ker}(T-\lambda \mathrm{id})^{r}=\operatorname{ker}\left(X_{2}^{2}-\lambda \mathrm{id}\right)^{r}$, where $r$ is the maximal size of $\lambda$-blocks in the Jordan normal form of $T$. Thus $V_{\lambda}^{T}$ is stable by $X_{2}$. It remains to show that it stable by $X_{1}$. By Lemma 2.9, if $u \in V_{\lambda}^{T}$ then

$$
(T-\lambda \mathrm{id})^{n} X_{1} u=X_{1} \sum_{j=0}^{n} \zeta_{j, n} S^{j}(T-\lambda \mathrm{id})^{n-j} u .
$$

By Lemma 2.1 of $[\mathbb{I}], S$ is nilpotent. Taking $n$ big enough, it follows that $(T-\lambda \mathrm{id})^{n} X_{1} u=0$ and whence $X_{1} u \in V_{\lambda}^{T}$.

Now Lemma 2.10 implies the next result.

Theorem 2.11. Let $\lambda_{1}, \ldots, \lambda_{t}$ be the different eigenvalues of $T$. Then $V$ decomposes into the direct sum of the $\mathcal{B}$-submodules $V_{\lambda_{i}}^{T}$.

In particular, if $V$ is indecomposable then $T$ has a unique eigenvalue. Hence either $X_{2}$ has a unique eigenvalue or else the eigenvalues of $X_{2}$ are $\lambda$ and $-\lambda$, with $\lambda \in \mathbb{k}^{\times}$.

Given $\lambda \in \mathbb{k}$, denote by $\mathcal{B}_{\lambda} \mathcal{M}_{\lambda}$ the full subcategory of ${ }_{\mathcal{B}} \mathcal{M}$ whose objects are the $\mathcal{B}$-modules $V$ such that $V=\operatorname{ker}(T-\lambda \text { id })^{m}$, for some $m \in \mathbb{N}_{0}$. With this notation, the next result follows immediately from Theorem 2.11.

Corollary 2.12. $\mathcal{B} \mathcal{M} \simeq \prod_{\lambda \in \mathbb{k}} \mathcal{B} \mathcal{M}_{\lambda}$.

The next result will be useful in $\S 3$.

Lemma 2.13. Let $\Lambda=\left\{v_{1}, \cdots, v_{n}\right\}$ be a basis of $V$ such that $\left[X_{1}\right]_{\Lambda}=E_{12}$ and $W$ a one-dimensional $\mathcal{B}$-submodule of $V$. Then:

(i) If $L$ is a complement (as a $\mathcal{B}$-module) of $W$ in $V$ then $L \cap V_{0}=\left\langle v_{1}\right\rangle$.

(ii) $W=\left\langle v_{1}\right\rangle$ does not have a complement (as a $\mathcal{B}$-submodule) in $V$.

Proof. (i) Assume that $W=\langle w\rangle$ and $\left\{u_{1}, u_{2}, \cdots, u_{n-1}\right\}$ is a basis of $L$. Since $W_{0}=W \cap V_{0} \neq 0$, it follows that $W \subset V_{0}$. Using that $v_{2}$ is a linear combination of $w, u_{1}, u_{2}, \cdots, u_{n-1}$ we see that $v_{1}=X_{1} v_{2} \in L$; hence $v_{1} \in V_{0} \cap L$.

(ii) It follows at once from (i) 


\section{INDECOMPOSABLE REPRESENTATIONS OF DIMENSION 2 AND 3}

3.1. Dimension 2. In this subsection we describe all 2-dimensional indecomposable representations of $\mathcal{B}$. Fix $(V, \rho)$ a 2-dimensional representation of $\mathcal{B}$.

Lemma 3.1. If $V \neq V_{0}$ then $V$ is indecomposable.

Proof. Suppose that $V$ is decomposable, i.e. there are non-trivial submodules $U$ and $W$ such that $V=U \oplus W$. Then $V_{0}=U_{0} \oplus W_{0}=U \oplus W=V$.

Define representations of $\mathcal{B}$ on the vector space $\mathbb{k}^{2}$ given by $X_{1}=E_{12}$ and the following action of $x_{2}$ :

$\diamond X_{2}=\left(\begin{array}{cc}a & b \\ 0 & a\end{array}\right), a, b \in \mathbb{k}$. This is denoted by $\mathcal{U}_{a, b}$.

$\diamond X_{2}=\left(\begin{array}{cc}a & 0 \\ 0 & -a\end{array}\right), a \in \mathbb{k}^{\times}$. This is denoted by $\mathcal{V}_{a}$.

It is easy to check that these are indecomposable modules pairwise nonisomorphic.

Theorem 3.2. Every 2-dimensional indecomposable representation of $\mathcal{B}$ is isomorphic either to $\mathcal{U}_{a, b}$, or to $\mathcal{V}_{a}$, or to $\mathbb{k}_{\lambda}^{2}$ for unique $a, b, \lambda \in \mathbb{k}$.

This confirms Theorem 2.11.

Proof. If $V=V_{0}$, then Proposition 2.8 applies. Assume that $V_{0} \neq 0$; then there exists a basis $\Lambda=\left\{v_{1}, v_{2}\right\}$ of $V$ such that $\left[X_{1}\right]_{\Lambda}=E_{12}$. Let $\left[X_{2}\right]_{\Lambda}=$ $\left(\begin{array}{ll}a & b \\ c & d\end{array}\right)$. Then (2.4) is satisfied if and only if

$$
c(a+d)=0 \quad \text { and } \quad d^{2}+c=a^{2} .
$$

Suppose that $c \neq 0$. Then by the first equation it follows that $d=-a$. Replacing in the second equation we have that $c=0$, which is a contradiction. Therefore $c=0$ and consequently $d=a$ or $d=-a$.

If $d=a$ then $V \simeq \mathcal{U}_{a, b}$. Assume that $d=-a \neq 0$ and take $w_{1}=v_{1}$ and $w_{2}=\frac{-b}{2 a} v_{1}+v_{2}$. Then $\Omega=\left\{w_{1}, w_{2}\right\}$ is a basis of $V$ such that $\left[X_{1}\right]_{\Omega}=E_{12}$ and $\left[X_{2}\right]_{\Omega}=\left(\begin{array}{cc}a & 0 \\ 0 & -a\end{array}\right)$. Thus $V \simeq \mathcal{V}_{a}$.

Corollary 3.3. If $\operatorname{Ext}^{1}\left(\mathbb{k}_{a}^{1}, \mathbb{k}_{b}^{1}\right) \neq 0$ then $a= \pm b$.

3.2. Dimension 3. Let $V$ be a $\mathcal{B}$-module of dimension 3 such that $V \neq V_{0}$. Throughout this subsection, $\Lambda=\left\{v_{1}, v_{2}, v_{3}\right\}$ denotes a basis of $V$ such that $\left[X_{1}\right]_{\Lambda}=E_{12}$. We define four families of representations of $\mathcal{B}$ on the vector 
space $V$ determined by the following action of $\left[X_{2}\right]_{\Lambda}$, for all $a, b, c, d, e \in \mathbb{k}$ :

$$
\begin{array}{ll}
\Theta_{1}:\left(\begin{array}{ccc}
a & b & c \\
0 & d & e \\
0 & \frac{a^{2}-d^{2}}{e} & -d
\end{array}\right), e \in \mathbb{k}^{\times} ; & \Theta_{2}:\left(\begin{array}{ccc}
a & b & c \\
0 & a & 0 \\
0 & d & e
\end{array}\right) ; \\
\Theta_{3}:\left(\begin{array}{ccc}
a & b & \frac{c^{2}-a^{2}}{d} \\
0 & c & 0 \\
d & e & -a
\end{array}\right), d \in \mathbb{k}^{\times} ; & \Theta_{4}:\left(\begin{array}{ccc}
a & b & c \\
0 & -a & 0 \\
0 & d & e
\end{array}\right), a \in \mathbb{k}^{\times} .
\end{array}
$$

Lemma 3.4. The families $\Theta_{1}, \Theta_{2}, \Theta_{3}$ and $\Theta_{4}$ contain all 3-dimensional representations of $\mathcal{B}$, up to isomorphism.

Proof. Let $\left[X_{2}\right]_{\Lambda}=\left(\begin{array}{ccc}\alpha & \beta & \gamma \\ \delta & \epsilon & \zeta \\ \eta & \theta & \iota\end{array}\right)$. Then (2.4) is valid if and only if

$$
\left\{\begin{array}{l}
\delta(\alpha+\epsilon)=-\zeta \eta \\
\zeta(\epsilon+\iota)=-\gamma \delta \\
\eta(\alpha+\iota)=-\delta \theta \\
\epsilon^{2}-\alpha^{2}=-\delta+\gamma \eta-\zeta \theta
\end{array}\right.
$$

Claim: If the system (3.1) has solution then $\delta=0$.

Assume that $\delta \neq 0$. If $\zeta=0$ then $\gamma=0$ and $\epsilon=-\alpha$. Thus, $\delta=0$ which is a contradiction. If $\zeta \neq 0$ then

$$
\gamma=\frac{-\zeta(\epsilon+\iota)}{\delta}, \quad \eta=\frac{-\delta(\alpha+\epsilon)}{\zeta} \text { and } \theta=\frac{(\alpha+\epsilon)(\alpha+\iota)}{\zeta} .
$$

From the last equation of (3.1), $\delta=0$ which is again a contradiction.

Assume $\delta=0$. Thus $\zeta \eta=0$. If $\zeta \neq 0$ then $\eta=0, \iota=-\epsilon$ and $\theta=\frac{\alpha^{2}-\epsilon^{2}}{\zeta}$. Hence $V$ belongs to the family $\Theta_{1}$. When $\zeta=0$ and $\eta \neq 0$, it follows that $\iota=-\alpha$ and $\gamma=\frac{\epsilon^{2}-\alpha^{2}}{\eta}$. Thus, $V$ belongs to the family $\Theta_{3}$. If $\zeta=0$ and $\eta=0$ then $\epsilon=|\alpha|$. In this case, $V$ belongs to the families $\Theta_{2}$ or $\Theta_{4}$.

Remark 3.5. Let $L$ a $\mathcal{B}$-submodule of $V$ of dimension 2 such that $L \cap V_{0}$ is one-dimensional. Fix $\bar{L}:=L /\left(L \cap V_{0}\right)=\langle\bar{u}\rangle$. Since $u \notin V_{0}$, we can suppose that $u=\alpha v_{1}+v_{2}+\gamma v_{3} \in L$, with $\alpha, \gamma \in \mathbb{k}$.

Proposition 3.6. Let $V$ be a $\mathcal{B}$-module. Then:

(i) the representations in the family $\Theta_{1}$ are always indecomposable;

(ii) a representation in the family $\Theta_{2}$ is indecomposable if and only if $c \neq 0$ and $e=a$ or $d \neq 0$ and $e=a$;

(iii) the representations in the family $\Theta_{3}$ are always indecomposable;

(iv) a representation in the family $\Theta_{4}$ is indecomposable if and only if $c \neq 0$ and $e=a$ or $d \neq 0$ and $e=-a$.

Proof. (i)] The unique one-dimensional $\mathcal{B}$-submodule of $V$ is $\left\langle v_{1}\right\rangle$ which does not have complement by Lemma 2.13 (ii). 
(ii) Let $V$ be a representation of $\mathcal{B}$ of the type $\Theta_{2}$. Suppose that $W=\langle w\rangle$ is a one-dimensional $\mathcal{B}$-submodule of $V$. Since $W \subset V_{0}$, see $\oint 2.4, w=$ $\alpha v_{1}+\beta v_{3}$, with $\alpha, \beta \in \mathbb{k}$. Note that $X_{2} w=\gamma w, \gamma \in \mathbb{k}$, if and only if $\beta(\gamma-e)=0$ and $\alpha(\gamma-a)=\beta c$. Consequently, the one-dimensional $\mathcal{B}$ submodules of $V$ are:

$\diamond\left\langle v_{1}\right\rangle,\left\langle v_{3}\right\rangle, c=0, e \neq a$,

$\diamond\left\langle\alpha v_{1}+\beta v_{3}\right\rangle, c=0, e=a$,

$\diamond\left\langle v_{1}\right\rangle,\left\langle v_{1}+\frac{e-a}{c} v_{3}\right\rangle, c \neq 0, e \neq a$

$\diamond\left\langle v_{1}\right\rangle, c \neq 0, e=a$.

Assume $e \neq a$. If $c \neq 0, V=\left\langle v_{1}+\frac{e-a}{c} v_{3}\right\rangle \oplus\left\langle v_{1}, v_{1}+v_{2}+\frac{d}{a-e} v_{3}\right\rangle$. If $c=0$, $V=\left\langle v_{3}\right\rangle \oplus\left\langle v_{1}, v_{1}+v_{2}+\frac{d}{a-e} v_{3}\right\rangle$. If $c=d=0, V=\left\langle v_{1}, v_{2}\right\rangle \oplus\left\langle v_{3}\right\rangle$. Hence, $V$ is decomposable.

Conversely, suppose $e=a$ and $c \neq 0$. Then the unique one-dimensional $\mathcal{B}$-submodule of $V$ is $\left\langle v_{1}\right\rangle$ which does not have complement. Suppose that $e=a$ and $d \neq 0$. Assume that $W$ is a one-dimensional $\mathcal{B}$-submodule of $V$ which admits a complement $L=\left\langle u_{1}, u_{2}\right\rangle$. Then by Lemma 2.13 (ii), $v_{1} \in L \cap V_{0}$. By Remark [3.5, $\bar{L}=\langle\bar{u}\rangle$ where $u=\alpha v_{1}+v_{2}+\beta v_{3}$. Thus $X_{2} \bar{u}=\gamma \bar{u}, \gamma \in \mathbb{k}$, if and only if $\gamma=a$ and $\beta(a-e)=d$. Since $d \neq 0$ and $e=a$ then $W$ does not have complement in $V$.

(iii) Suppose that $W=\langle w\rangle$ is a one-dimensional $\mathcal{B}$-submodule of $V$ which admits a complement $L$. Then by Lemma 2.13 (ii), $\left\langle v_{1}\right\rangle=L \cap V_{0}$, which is a contradiction because $d \neq 0$.

(iv): Analogous to item (ii).

3.2.1. Isomorphism classes in $\Theta_{1}$. Assume $V$ in the family $\Theta_{1}$. We distinguish: for all $a, b, c, d, e \in \mathbb{k}$

$\diamond X_{2}=\left(\begin{array}{ccc}a & b & c \\ 0 & d & e \\ 0 & \frac{a^{2}-d^{2}}{e} & -d\end{array}\right), e \in \mathbb{k}^{\times}$. This is denoted by $\mathcal{Y}_{a, b, c, d, e}$.

$\diamond X_{2}=\left(\begin{array}{ccc}a & b & 0 \\ 0 & a & 1 \\ 0 & 0 & -a\end{array}\right)$. This is denoted by $\mathcal{U}^{a, b}$.

By Proposition 3.6 (i), these representations are indecomposable. Note that $\mathcal{U}^{a, b}=\mathcal{Y}_{a, b, 0, a, 1}$.

Proposition 3.7. Every 3-dimensional indecomposable representation $V$ of $\mathcal{B}$ in $\Theta_{1}$ is isomorphic either to $\mathcal{U}^{a, b}$, or to $\mathcal{Y}_{a, b, c, d, e}$. Moreover,

$\mathcal{Y}_{a, b, c, d, e} \simeq \mathcal{Y}_{a, b^{\prime}, c^{\prime}, d^{\prime}, e^{\prime}}$ if and only if $\left(a-d^{\prime}\right) \frac{c e^{\prime}-c^{\prime} e}{e^{\prime}}=e\left(b^{\prime}-b\right)+c\left(d^{\prime}-d\right)$.

In particular, $\mathcal{U}^{a, b} \simeq \mathcal{U}^{a, b^{\prime}}$ if and only if $b=b^{\prime}$.

Proof. Since $\left\langle X_{2} v_{1}\right\rangle=\operatorname{Im} X_{1}$, we obtain that $a$ is invariant. Consider the indecomposable representation $\mathcal{Y}_{a, b^{\prime}, c^{\prime}, d^{\prime}, e^{\prime}}$ of $\mathcal{B}$. If $d^{\prime}=a$, taking the basis $\left\{v_{1}, \frac{c^{\prime}}{e^{\prime}} v_{1}+v_{2}, \frac{1}{e^{\prime}} v_{3}\right\}$ we conclude that $\mathcal{Y}_{a, b^{\prime}, c^{\prime}, d^{\prime}, e^{\prime}} \simeq \mathcal{U}^{a, b^{\prime}}$. 
Note that $\mathcal{Y}_{a, b, c, d, e}$ and $\mathcal{Y}_{a, b^{\prime}, c, d^{\prime}, e^{\prime}}$ are isomorphic if and only if there exists a basis $\left\{w_{1}, w_{2}, w_{3}\right\}$ of $V$ such that $X_{1} w_{1}=X_{1} w_{3}=0, X_{1} w_{2}=w_{1}, X_{2} w_{1}=$ $a w_{1}, X_{2} w_{2}=b^{\prime} w_{1}+d^{\prime} w_{2}+\frac{a^{2}-d^{\prime 2}}{e^{\prime}} w_{3}$ and $X_{2} w_{3}=c^{\prime} w_{1}+e^{\prime} w_{2}-d^{\prime} w_{3}$. Since $\left\langle v_{1}\right\rangle=\operatorname{Im} X_{1}$ and $V_{0}$ has dimension 2, then we can consider $w_{1}=v_{1}$, $w_{2}=\lambda_{1} v_{1}+\lambda_{2} v_{2}+\lambda_{3} v_{3}$ and $w_{3}=\beta_{1} v_{1}+\beta_{3} v_{3}, \lambda_{1}, \lambda_{2}, \lambda_{3}, \beta_{1}, \beta_{3} \in \mathbb{k}$. Then, $\mathcal{Y}_{a, b, c, d, e} \simeq \mathcal{Y}_{a, b^{\prime}, c, d^{\prime}, e^{\prime}}$ if and only if $\left(a-d^{\prime}\right) \frac{c e^{\prime}-c^{\prime} e}{e^{\prime}}=e\left(b^{\prime}-b\right)+c\left(d^{\prime}-d\right)$.

3.2.2. Isomorphism classes in $\Theta_{2}$. Consider $V$ in the family $\Theta_{2}$ and the following distinguish representations: for all $a \in \mathbb{k}$

$\diamond X_{2}=\left(\begin{array}{lll}a & 0 & 0 \\ 0 & a & 0 \\ 0 & 1 & a\end{array}\right)$. This is denoted by $\mathcal{R}_{a}$.

$\diamond X_{2}=\left(\begin{array}{ccc}a & 0 & 1 \\ 0 & a & 0 \\ 0 & 0 & a\end{array}\right)$. This is denoted by $\mathcal{S}_{a}$.

$\diamond X_{2}=\left(\begin{array}{ccc}a & 0 & b \\ 0 & a & 0 \\ 0 & c & a\end{array}\right), b \in \mathbb{k}^{\times}$or $c \in \mathbb{k}^{\times}$. This is denoted by $\mathcal{T}_{a, b, c}$.

By Proposition 3.6 (ii), these are indecomposable representations. Notice that $\mathcal{R}_{a}=\mathcal{T}_{a, 0,1}$ and $\mathcal{S}_{a}=\mathcal{T}_{a, 1,0}$.

Proposition 3.8. Every 3-dimensional indecomposable representation $V$ of $\mathcal{B}$ in $\Theta_{2}$ is isomorphic either to $\mathcal{R}_{a}$, or to $\mathcal{S}_{a}$ or to $\mathcal{T}_{a, b, c}$. Moreover, $\mathcal{T}_{a, b, c}$ and $\mathcal{T}_{a, b^{\prime}, c^{\prime}}$ are isomorphic if and only if $b c=b^{\prime} c^{\prime}$.

Proof. Let $V^{\prime}$ the representation of $\mathcal{B}$ given by

$$
\left[X_{2}\right]_{\Lambda}=\left(\begin{array}{ccc}
a & d^{\prime} & b^{\prime} \\
0 & a & 0 \\
0 & c^{\prime} & a
\end{array}\right)
$$

If $b^{\prime}=0$, then by Proposition 3.6 (ii) we have that $c^{\prime} \neq 0$. In this case, taking the basis $\left\{v_{1}, v_{2}, d^{\prime} v_{1}+c^{\prime} v_{3}\right\}$ of $V^{\prime}$, we conclude that $V^{\prime} \simeq \mathcal{R}_{a}$. Similarly, if $c^{\prime}=0$ then $b^{\prime} \neq 0$. Taking the basis $\left\{v_{1}, v_{2}-\frac{d^{\prime}}{b^{\prime}} v_{3}, \frac{1}{b^{\prime}} v_{3}\right\}$ of $V^{\prime}$, we obtain that $V^{\prime} \simeq \mathcal{S}_{a}$. If $b, b^{\prime}, c, c^{\prime} \in \mathbb{k}^{\times}$, taking the basis $\left\{v_{1}, v_{2}, \frac{d^{\prime}}{c^{\prime}} v_{1}+v_{3}\right\}$, it follows that $V^{\prime} \simeq \mathcal{T}_{a, b^{\prime}, c^{\prime}}$.

Finally, notice that $\mathcal{T}_{a, b, c} \simeq \mathcal{T}_{a, b^{\prime}, c^{\prime}}$ if and only if there exists a basis $\left\{w_{1}, w_{2}, w_{3}\right\}$ of $\mathbb{k}^{3}$ such that $X_{1} w_{1}=X_{1} w_{3}=0, X_{1} w_{2}=w_{1}, X_{2} w_{1}=a w_{1}$, $X_{2} w_{2}=a w_{2}+c w_{3}$ and $X_{2} w_{3}=b w_{1}+a w_{3}$. We can assume $w_{1}=v_{1}$, $w_{2}=\lambda_{1} v_{1}+\lambda_{2} v_{2}+\lambda_{3} v_{3}$ and $w_{3}=\beta_{1} v_{1}+\beta_{3} v_{3}, \lambda_{1}, \lambda_{2}, \lambda_{3}, \beta_{1}, \beta_{3} \in \mathbb{k}$. Note that $X_{2} w_{2}=w_{1}$ if and only if $\lambda_{2}=1$. Moreover, $X_{2} w_{2}=a w_{2}+c w_{3}$ and $X_{2} w_{3}=b w_{1}+a w_{3}$ if and only if $b c=b^{\prime} c^{\prime}$.

3.2.3. Isomorphism classes in $\Theta_{3}$. Consider $V$ in the family $\Theta_{3}$ and the following distinguished representations: for all $a, b, c, d, e \in \mathbb{k}$ 
$\diamond X_{2}=\left(\begin{array}{ccc}a & b & \frac{c^{2}-a^{2}}{d} \\ 0 & c & 0 \\ d & e & -a\end{array}\right), d \in \mathbb{k}^{\times}$. This is denoted by $\mathcal{W}_{a, b, c, d, e}$.

$\diamond X_{2}=\left(\begin{array}{ccc}a & b & 0 \\ 0 & a & 0 \\ 1 & 0 & -a\end{array}\right)$. This is denoted by $\mathcal{U}_{a, b}$.

By Proposition 3.6 (iii), these representations are indecomposable. Observe that $\mathcal{U}_{a, b}=\mathcal{W}_{a, b, a, 1,0}$

Proposition 3.9. Every 3-dimensional indecomposable representation $V$ of $\mathcal{B}$ in $\Theta_{3}$ is isomorphic either to $\mathcal{U}_{a, b}$, or to $\mathcal{W}_{a, b, c, d, e}$. Moreover,

$$
\mathcal{W}_{a, b, c, d, e} \simeq \mathcal{W}_{a^{\prime}, b^{\prime}, c, d^{\prime}, e^{\prime}} \text { if and only if } \frac{a e-b d-c e}{d}=\frac{a^{\prime} e^{\prime}-b^{\prime} d^{\prime}-c e^{\prime}}{d^{\prime}} \text {. }
$$

In particular, $\mathcal{U}_{a, b} \simeq \mathcal{U}_{a, b^{\prime}}$ iff $b=b^{\prime}$.

Proof. Since the characteristic polynomial of $X_{2}$ is $(t-c)^{2}(t+c), c$ is an invariant. Let the indecomposable representation $\mathcal{W}_{a^{\prime}, b^{\prime}, c, d^{\prime}, e^{\prime}}$ of $\mathcal{B}$. If $c=a$, taking the basis $\left\{v_{1},-\frac{e}{d} v_{1}+v_{2}, d v_{3}\right\}$ we conclude that $\mathcal{W}_{a^{\prime}, b^{\prime}, c, d^{\prime}, e^{\prime}} \simeq \mathcal{U}_{a^{\prime}, b^{\prime}}$. Note that $\mathcal{W}_{a, b, c, d, e} \simeq \mathcal{W}_{a^{\prime}, b^{\prime}, c, d^{\prime}, e^{\prime}}$ if and only if there is a basis $\left\{w_{1}, w_{2}, w_{3}\right\}$ of $\mathbb{k}^{3}$ such that $X_{1} w_{1}=X_{1} w_{3}=0, X_{1} w_{2}=w_{1}, X_{2} w_{1}=a w_{1}, x_{2} w_{2}=a w_{2}+c w_{3}$ and $X_{2} w_{3}=b w_{1}+a w_{3}$. We can assume $w_{1}=v_{1}, w_{2}=\lambda_{1} v_{1}+\lambda_{2} v_{2}+\lambda_{3} v_{3}$ and $w_{3}=\beta_{1} v_{1}+\beta_{3} v_{3}$, where $\lambda_{1}, \lambda_{2}, \lambda_{3}, \beta_{1}, \beta_{3} \in \mathbb{k}$. However $X_{2} w_{1}=a^{\prime} w_{1}+d^{\prime} w_{3}$ if and only if $\beta_{1}=\frac{a-a^{\prime}}{d^{\prime}}$ e $\beta_{3}=\frac{d}{d^{\prime}}$. With this choose of $\beta_{1}$ and $\beta_{3}$ we have that $X_{2} w_{3}=\frac{c^{2}-a^{\prime 2}}{d^{\prime}} w_{1}-a^{\prime} w_{3}$. Finally, $X_{2} w_{2}=b_{1} w_{1}+c w_{2}+e^{\prime} w_{3}$ if and only if $\frac{a e-b d-c e}{d}=\frac{a^{\prime} e^{\prime}-b^{\prime} d^{\prime}-c e^{\prime}}{d^{\prime}}$.

3.2.4. Isomorphism classes in $\Theta_{4}$. Consider $V$ in $\Theta_{4}$ and the following distinguish representations: for all $a \in \mathbb{k}^{\times}$

$\diamond X_{2}=\left(\begin{array}{ccc}a & 0 & 1 \\ 0 & -a & 0 \\ 0 & 0 & a\end{array}\right)$. This is denoted by $\mathcal{V}^{a}$.

$\diamond X_{2}=\left(\begin{array}{ccc}a & 0 & 0 \\ 0 & -a & 0 \\ 0 & 1 & -a\end{array}\right)$. This is denoted by $\mathcal{V}_{a}$.

By Proposition 3.6 (iv), these are indecomposable representations pairwise non-isomorphic.

Proposition 3.10. Every 3-dimensional indecomposable representation $V$ of $\mathcal{B}$ in $\Theta_{4}$ is isomorphic either to $\mathcal{V}^{a}$ or to $\mathcal{V}_{a}$ for unique $a \in \mathbb{k}^{\times}$.

Proof. Let $V^{\prime}$ be a 3-dimensional indecomposable representation of $\mathcal{B}$ such that

$$
\left[X_{2}\right]_{\Lambda}=\left(\begin{array}{ccc}
a & b & c \\
0 & -a & 0 \\
0 & d & e
\end{array}\right), a \in \mathbb{k}^{\times}
$$


Since $V^{\prime}$ is indecomposable, by Proposition 3.6 (iv) we have that $c \neq 0$ and $e=a$ or $d \neq 0$ and $e=-a$. If $c \neq 0$ and $e=a$, taking the basis $\left\{v_{1}, \frac{c d-2 a b}{4 a^{2}} v_{1}+v_{2}-\frac{d}{2 a} v_{3}, v_{1}+\frac{1}{c} v_{3}\right\}$ of $V^{\prime}$, we obtain $V^{\prime} \simeq \mathcal{V}^{a}$. If $d \neq 0$ and $e=-a$, taking the basis $\left\{v_{1},-\frac{2 a b+c d}{4 a^{2}} v_{1}+v_{2},-\frac{d c}{2 a} v_{1}+d v_{3}\right\}$ of $V^{\prime}$, it follows that $V^{\prime} \simeq \mathcal{V}_{a}$.

\subsubsection{Classification of indecomposable 3-dimensional $\mathcal{B}$-modules.}

Theorem 3.11. Every 3-dimensional indecomposable $\mathcal{B}$-module is isomorphic either to $\mathbb{K}_{\lambda}^{3}$ for a unique $\lambda$, or else to a representation in one of the families $\Theta_{j}, j=1,2,3,4$, with the constraints described in Proposition [3.6. The isomorphism classes are described in Propositions [3.7. 3.8, 3.9 and 3.10.

Again, this agrees with Theorem 2.11.

Remark 3.12. It is straightforward to verify that two 3-dimensional indecomposable representations of $\mathcal{B}$ that belong to different families $\Theta_{i}, i=1,2,3,4$, are not isomorphic.

\section{Families of indecomposable $\mathcal{B}$-Modules}

Throughout this section $(V, \rho)$ is an $n$-dimensional representation of $\mathcal{B}$, $\Lambda=\left\{v_{1}, \ldots, v_{n}\right\}$ is a basis of $V, X_{1}=\rho\left(x_{1}\right), X_{2}=\rho\left(x_{2}\right)$ and $\left[X_{1}\right]_{\Lambda}=E_{12}$.

4.1. The family $\mathcal{U}_{a}$. Let $a \in \mathbb{k}$. Consider the following action of $X_{2}$ on $V$ :

$$
\left[X_{2}\right]_{\Lambda}=\left(\begin{array}{ccccccc}
a & 0 & 0 & 0 & \ldots & 0 & 0 \\
0 & a & 0 & 0 & \ldots & 0 & 0 \\
0 & 1 & a & 0 & \ldots & 0 & 0 \\
0 & 0 & 1 & a & \ldots & 0 & 0 \\
\vdots & \vdots & \vdots & \ddots & \ddots & \vdots & \vdots \\
0 & 0 & 0 & \ldots & 1 & a & 0 \\
0 & 0 & 0 & \ldots & 0 & 1 & a
\end{array}\right)
$$

Clearly $V$ with this action is a $\mathcal{B}$-module which will be denoted by $\mathcal{U}_{a}$.

Lemma 4.1. Let $\mathcal{W}$ be a proper $\mathcal{B}$-submodule of $\mathcal{U}_{a}$. Then:

(i) $v_{2} \notin \mathcal{W}$;

(ii) If $v=\sum_{i=1}^{n} \lambda_{i} v_{i} \in \mathcal{W}$ then $\lambda_{2}=0$.

Proof. (i) Suppose $v_{2} \in \mathcal{W}$. Then $v_{1}=X_{1} v_{2} \in \mathcal{W}$ and $X_{2} v_{2}=a v_{2}+v_{3} \in$ $\mathcal{W}$. Hence $v_{3} \in \mathcal{W}$. Again, $X_{2} v_{3}=a v_{3}+v_{4} \in \mathcal{W}$ and consequently $v_{4} \in \mathcal{W}$. With this procedure, we obtain that $\Lambda \subset \mathcal{W}$. Thus, $\mathcal{W}=\mathcal{U}_{a}$ and we have a contradiction.

(ii) Assume $\lambda_{2} \neq 0$ and fix $w_{1}=\lambda_{2}^{-1} v$. Thus $w_{1}=\alpha_{1} v_{1}+v_{2}+\ldots+\alpha_{n} v_{n}$, where $\alpha_{i}=\lambda_{2}^{-1} \lambda_{i}$, for all $1 \leq i \leq n$. Consider the following elements of $V$ :

$$
w_{j}:=v_{j+1}+\alpha_{3} v_{j+2}+\ldots+\alpha_{n-j+1} v_{n}, \quad \text { for all } 2 \leq j \leq n-2 .
$$


By a straightforward calculation, we obtain that $X_{2} w_{j}=a w_{j}+w_{j+1}$, for all $1 \leq j \leq n-2$. Thus, $w_{1}, \ldots, w_{n-2} \in \mathcal{W}$ and $X_{2} w_{n-2}=a w_{n-2}+v_{n}$. Therefore, $v_{n} \in \mathcal{W}$. But $w_{n-2}=v_{n-1}+\alpha_{3} v_{n}$ and whence $v_{n-1} \in \mathcal{W}$. By this procedure, it follows that $v_{3}, \ldots, v_{n} \in \mathcal{W}$. From $v_{1}=X_{1} w_{1} \in \mathcal{W}$, it follows that $v_{2} \in \mathcal{W}$ which contradicts (i).

Theorem 4.2. $\mathcal{U}_{a}$ is an indecomposable $\mathcal{B}$-module, for all $n \geq 2$.

Proof. Suppose $\mathcal{U}_{a}$ decomposable. Let $\mathcal{W}, \widetilde{\mathcal{W}}$ be nontrivial $\mathcal{B}$-submodules of $\mathcal{U}_{a}$ such that $\mathcal{U}_{a}=\mathcal{W} \oplus \widetilde{\mathcal{W}}$. Consider $\left\{w_{1}, \ldots, w_{r}\right\}$ and $\left\{w_{r+1}, \ldots, w_{n}\right\}$ basis of $\mathcal{W}$ and $\widetilde{\mathcal{W}}$ respectively. By Lemma 4.1, $w_{i}=\lambda_{i 1} v_{1}+\lambda_{i 3} v_{3}+\ldots+\lambda_{i n} v_{n}$ for all $1 \leq i \leq n$. Since $v_{2} \in \mathcal{U}_{a}$, there exist $\alpha_{1}, \ldots, \alpha_{n} \in \mathbb{k}$ such that $v_{2}=\alpha_{1} w_{1}+\ldots+\alpha_{n} w_{n}$, a contradiction.

4.2. The family $\mathcal{V}_{a}$. Let $a \in \mathbb{k}^{\times}$. Consider the following action of $X_{2}$ on $V:$

$$
\left[X_{2}\right]_{\Lambda}=\left(\begin{array}{ccccccc}
a & 0 & 0 & 0 & \ldots & 0 & 0 \\
0 & -a & 0 & 0 & \ldots & 0 & 0 \\
0 & 1 & -a & 0 & \ldots & 0 & 0 \\
0 & 0 & 1 & -a & \ldots & 0 & 0 \\
\vdots & \vdots & \vdots & \ddots & \ddots & \vdots & \vdots \\
0 & 0 & 0 & \ldots & 1 & -a & 0 \\
0 & 0 & 0 & \ldots & 0 & 1 & -a
\end{array}\right) .
$$

Notice that $V$ is a $\mathcal{B}$-module which will be denoted by $\mathcal{V}_{a}$. Since $a \neq 0$, $\mathcal{U}_{a}$ and $\mathcal{V}_{a}$ are not isomorphic.

Theorem 4.3. $\mathcal{V}_{a}$ is an indecomposable $\mathcal{B}$-module, for all $n \geq 2$.

Proof. Let $\mathcal{W}$ a proper $\mathcal{B}$-submodule of $\mathcal{V}_{a}$. As in Lemma 4.1 (i), we can show that $v_{2} \notin \mathcal{W}$. Let $v \in \mathcal{W}$ such that $v=\sum_{i=1}^{n} \lambda_{i} v_{i}$. Assume that $\lambda_{2} \neq 0$ and consider $u:=\lambda_{2}^{-1} v \in \mathcal{W}$. Then $v_{1}=X_{1} u \in \mathcal{W}$. Take $w_{1}:=u-\lambda_{2}^{-1} \lambda_{1} v_{1}$ and note that $w_{1}=\alpha_{2} v_{2}+\ldots+\alpha_{n} v_{n}$, where $\alpha_{i}=\lambda_{2}^{-1} \lambda_{i}$, for all $2 \leq i \leq n$. Considering the following elements of $V$

$$
w_{j}:=v_{j+1}+\alpha_{3} v_{j+2}+\ldots+\alpha_{n-j+1} v_{n}, \quad \text { for all } 2 \leq j \leq n-2,
$$

it follows $X_{2} w_{j}=-a w_{j}+w_{j+1}$, for all $1 \leq j \leq n-2$. As in Lemma 4.1, this implies that $v_{2} \in \mathcal{W}$ which is a contradiction. Thus, the result follows as in Theorem 4.2.

\section{REFERENCES}

[AAH1] N. Andruskiewitsch, I. Angiono and I. Heckenberger. Liftings of Jordan and super Jordan planes. Proc. Edinb. Math. Soc., II. Ser., to appear. arXiv:1512.09271.

[AAH2] N. Andruskiewitsch, I. Angiono and I. Heckenberger. On finite GK-dimensional Nichols algebras over abelian groups. arXiv: 1606.02521.

[AS] Artin, M., Schelter, W. F. Graded algebras of global dimension 3. Adv. in Math. 66 171-216 (1987). 
[G] D. I. Gurevich, The Yang-Baxter equation and the generalization of formal Lie theory. Dokl. Akad. Nauk SSSR. 288 (4), 797-801 (1986).

[I] N. Iyudu, Representation Spaces of the Jordan Plane. Comm. Algebra. 42 (8), 3507-3540 (2014).

[PP] A. Polishchuk and L. Positselski, Quadratic algebras. University Lecture Series AMS 37, (2005).

FamaF-Universidad Nacional de Córdoba, Ciem (COniCET), Medina Allende s/n, Ciudad Universitaria, 5000 Córdoba, República Argentina.

E-mail address: andrus@famaf.unc.edu.ar

Departamento de Matemática, Universidade Federal de Santa Maria, 97105900, SANTA MARIA, RS, BRAZIL

E-mail address: bagio@smail.ufsm.br, saradia.flora@ufsm.br, flores@ufsm.br 